\title{
STOMACH
}

\section{Effect of Helicobacter pylori eradication on development of dyspeptic and reflux disease in healthy asymptomatic subjects}

\author{
D Vaira, N Vakil, M Rugge, L Gatta, C Ricci, M Menegatti, G Leandro, J Holton, V M Russo, M Miglioli
}

See end of article for authors' affiliations

.....................

Correspondence to: Professor D Vaira, Department of Internal Medicine and Gastroenterology, University of Bologna, S Orsola Hospital-Nuove Patologie, Via Massarenti, 9, 40138 Bologna, Italy; vairadin@med.unibo.it

Accepted for publication 25 June 2003

\begin{abstract}
Background and aim: There are few data on the course of Helicobacter pylori infection in asymptomatic subjects. The aim of this study was to assess the effect of eradication therapy on the development of dyspeptic and gastro-oesophageal reflux disease in a cohort of asymptomatic individuals observed over a prolonged period.

Methods: A total of 169 blood donors infected with $H$ pylori who had volunteered for studies on eradication in 1990 formed the cohort. To be included in this cohort subjects had to have no symptoms, as determined by a validated symptom questionnaire at the baseline visit. Eighty eight subjects were infected with $H$ pylori while 81 had successfully undergone eradication therapy. Subjects were followed up (annually) using the same symptom questionnaire and in 2000 they underwent repeat endoscopy.

Results: Thirteen subjects developed symptoms during follow up. The incidence of symptoms in $H$ pylori positive subjects was $1.893 / 100$ person-years of follow up and in $H$ pylori negative individuals 0.163 / 100 person-years of follow up. $H$ pylori infected subjects were significantly more likely to develop symptoms ( $\log$ rank test, $p=0.003$ ) as well as those infected with CagA positive strains (log rank test, $p=0.017)$. The development of symptomatic gastro-oesophageal reflux disease was no different in individuals with and without eradication (odds ratio 0.57 (95\% confidence interval 0.26-1.24); $\mathrm{p}=0.163)$.

Conclusions: $H$ pylori eradication prevents the development of dyspeptic symptoms and peptic ulcer disease in healthy asymptomatic blood donors and is not associated with an increase in the incidence of symptomatic gastro-oesophageal reflux disease.
\end{abstract}

$\mathrm{H}$ elicobacter pylori is a human pathogen that causes gastritis, peptic ulcer disease, and is recognised as a class 1 gastric carcinogen. ${ }^{1}$ It is well established that eradication of $H$ pylori heals duodenal ulcers and prevents recurrences of peptic ulcer disease. ${ }^{2}$ However, there is controversy about the benefit of $H$ pylori eradication in some clinical conditions, such as non-ulcer dyspepsia, gastrooesophageal reflux disease (GORD), and patients taking chronic non-steroidal anti-inflammatory drugs (NSAIDs). ${ }^{3-6}$ Moreover, there are few data on the course of the infection in asymptomatic subjects. It has recently been established that chronic $H$ pylori infection may lead to gastric atrophy and intestinal metaplasia, which are significant risk factors for the development of gastric cancer, ${ }^{7}$ and eradication therapy may prevent this progression. Despite this, current guidelines do not recommend eradication therapy in asymptomatic subjects, reflecting the controversy in this area. ${ }^{8}$

We performed a long term prospective study to investigate the development of dyspeptic symptoms and GORD in a population of asymptomatic $H$ pylori positive subjects who underwent successful eradication of $H$ pylori and compared them with a similar group that remained infected with $H$ pylori. We also sought to examine the natural history of $H$ pylori infection in subjects infected with cytotoxin associated gene A (CagA) positive strains compared with subjects infected with CagA negative strains.

\section{METHODS}

\section{Cohort}

In 1990-92, we performed a study on the endoscopic prevalence of gastroduodenal diseases in 276 asymptomatic Caucasian blood donors infected with $H$ pylori referred to the transfusion unit in Bologna, Italy (first donor endoscoped in 1992, last in 1993). Details of this study have been published elsewhere. ${ }^{910}$ There was no consensus at that time on whether $H$ pylori eradication therapy should be administered to asymptomatic individuals and there is none today. Following the conclusion of that study, all individuals received eradication therapy. A variety of regimens were used which would be considered ineffective today but were in general use at that time. We decided to perform a long term natural history study in this study population and offered entry to all subjects who participated in the original study. The present study is a long term case control study of a cohort of asymptomatic blood donors with persistent $H$ pylori infection compared with asymptomatic blood donors who had successful eradication of $H$ pylori.

A total of 169 blood donors infected with $H$ pylori who had volunteered for studies on eradication in 1990-1992, and who agreed to take part in this study, formed the cohort. To be included in this cohort, subjects had to have no symptoms, as determined by a validated symptom questionnaire at the baseline visit performed after treatment in 1992. Success or failure of eradication therapy was determined by endoscopic tests performed at least four weeks after the end of treatment. Two biopsies were taken from the antrum for histology (haematoxylin-eosin and Giemsa stain), one sample from the antrum was obtained for culture (performed

Abbreviations: GORD, gastro-oesophageal reflux disease; NSAIDs, non-steroidal anti-inflammatory drugs; OR, odds ratio; CagA, cytotoxin associated gene $\mathrm{A}$ 
on selective blood agar), and one sample was obtained from the antrum for the rapid urease test. The endoscopic examinations were performed by an investigator blinded to the $H$ pylori status of the patient. Rapid urease tests were performed by nursing staff and results were not communicated to the endoscopist.

Subjects were classified as being infected with $H$ pylori at baseline if the rapid urease test and histology were positive and/or if culture of gastric biopsy specimens was positive. All other patients were classified as negative.

At inclusion in this study, 88 subjects were infected with $H$ pylori while 81 were $H$ pylori negative.

\section{Baseline visit}

At the baseline visit, subjects completed a symptom questionnaire that has been validated in Italian subjects by the Italian Dyspepsia Study Group and measures a number of dyspeptic and GORD related symptoms. ${ }^{11-13}$ Serum samples were obtained and analysed later for the anti-CagA antibody by western blot. The characteristics of the cohort are shown in table 1.

\section{Annual follow up examinations}

Subjects returned annually to complete the same validated symptom questionnaire used at baseline. They underwent a physical examination and a review of any medications taken for dyspeptic symptoms in the previous year (NSAIDs, proton pump inhibitors, $\mathrm{H}_{2}$ receptor antagonists, antibiotics). Subjects developing dyspeptic or GORD symptoms during the period between the annual visits were asked to report immediately and underwent endoscopy and treatment according to the findings.

\section{Final visit}

In 2000, after a mean follow up of 8.5 years (range 6.2-9.3), subjects returned for the final visit. They underwent a physical examination and endoscopy, and completed the validated symptom questionnaire. Medication use, tests, and procedures performed in the previous year were reviewed. At endoscopy, six biopsy samples were obtained. Two biopsies were taken from the antrum and two from the corpus for histology (haematoxylin-eosin and Giemsa stain), one sample from the antrum was obtained for culture (performed on selective blood agar), and one sample was obtained from the antrum for the rapid urease test. Endoscopies were performed by a single investigator who was blinded to the $H$ pylori status of the patient. Rapid urease tests were performed by nursing staff and the results were not communicated to the endoscopist. Patients were classified as being infected with $H$ pylori if the rapid urease test and histology were positive and/or if culture of gastric biopsy specimens was positive. All other patients were classified as negative. ${ }^{14}$

Table 1 Characteristics of asymptomatic subjects forming the cohort

\begin{tabular}{lll}
\hline & $\begin{array}{l}\text { H pylori positive } \\
(\mathbf{n}=\mathbf{8 8})\end{array}$ & $\begin{array}{l}\boldsymbol{H} \text { pylori eradicated } \\
(\mathbf{n}=\mathbf{8 1})\end{array}$ \\
\hline Sex (M/F) & $57 / 31$ & $56 / 25$ \\
Age (y) (mean (SD)) & $47(11)$ & $47(12)$ \\
Alcohol use & 17 & 15 \\
Smokers & 6 & 9 \\
Chronic NSAID intake & 0 & 0 \\
Race & All Caucasian & All Caucasian \\
\hline
\end{tabular}

NSAID, non-steroidal anti-inflammatory drug.

\section{Anti-CagA antibody determination}

CagA status was determined by western blot using the serum sample obtained at baseline. A whole cell suspension of $H$ pylori CCUG 17874 (CagA positive type strain) was denaturised in Laemmli's solution at $100^{\circ} \mathrm{C}$ for 10 minutes, and run electrophoretically in a $10 \%$ sodium dodecyl sulphatepolyacrylamide gel. Separated proteins were transferred to nitrocellulose which was saturated for 30 minutes with a 3\% defatted milk solution in phosphate saline buffer, $\mathrm{pH} 7.5$, containing $0.1 \%$ Triton $\mathrm{X}$ (MTB). Nitrocellulose strips were incubated with serum samples diluted 1:1000 in MTB at room temperature overnight. After washing with MTB, strips were incubated with antihuman immunoglobulin G conjugated with peroxidase at room temperature for 90 minutes. The strips were washed and the reaction was visualised by an enhanced chemiluminescence assay (Amersham, Pharmacia Biotech, UK). As controls, the following sera kindly provided by Drs Covacci, Telford, and Burroni (IRIS-Biocine, Siena, Italy) were used: antirecombinant CagA, antirecombinant VacA, antipurified urease, and heat shock protein. Subjects were considered to be CagA+ if more than four bands of reaction were evident in the blots. ${ }^{15}$

\section{Statistical analysis}

The principal end point of the study was the development of symptoms, and subjects developing symptoms left the study as they had reached the predefined end point. Statistical tests used were Fisher's exact test (two tailed) for frequencies and the Mann-Whitney rank sum test or the Kruskal-Wallis test for means. The cumulative percentage of symptom development was analysed by means of life tables. Product limit

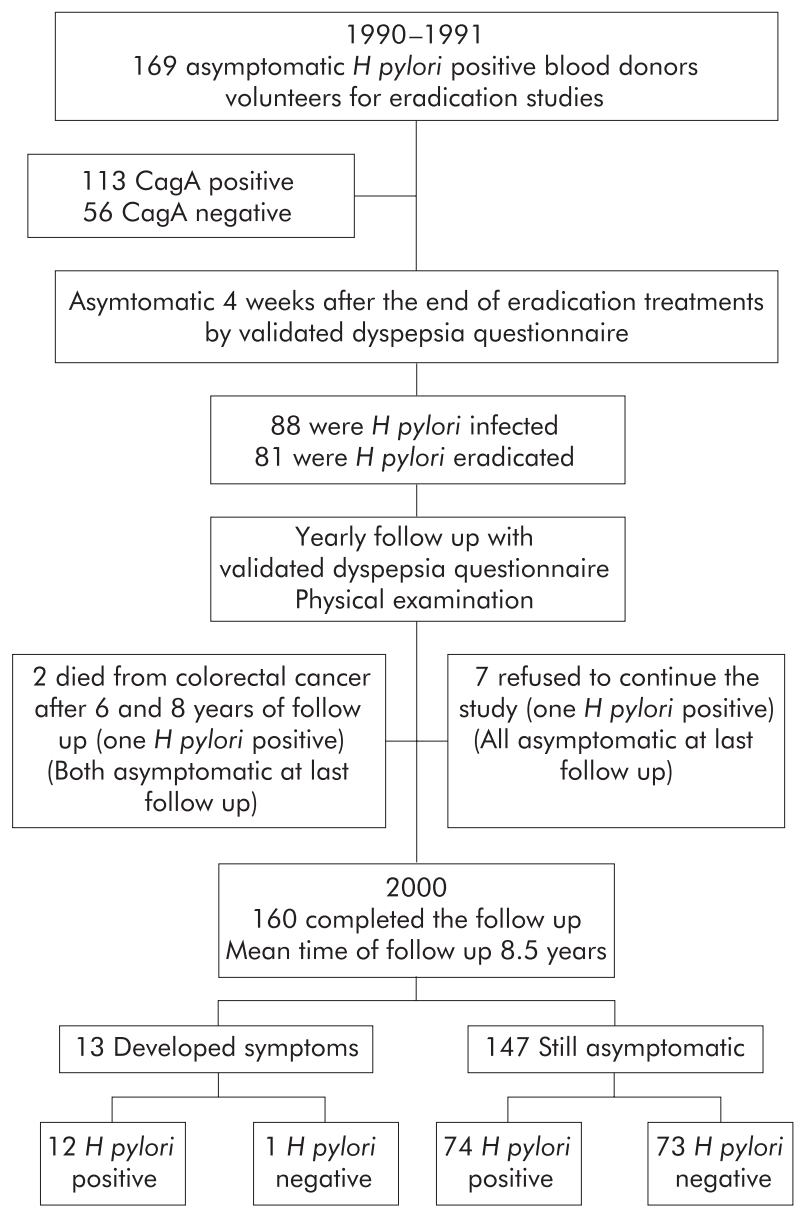

Figure 1 Flow chart of the study. 
Table 2 Endoscopic findings in 147 blood donors who completed follow up

\begin{tabular}{lll}
\hline & $\begin{array}{l}\boldsymbol{H} \text { pylori positive } \\
(\mathbf{n = 7 4 )}\end{array}$ & $\begin{array}{l}\boldsymbol{H} \text { pylori eradicated } \\
(\mathbf{n}=73)\end{array}$ \\
\hline Duodenal ulcer & $8(10.8 \% ; 5.6-19.9)$ & 0 \\
Gastric ulcer & $1(1.4 \% ; 0.2-7.3)$ & $1(1.4 \% ; 0.2-7.4)$ \\
Erosive duodenitis & $7(9.5 \% ; 4.7-18.3)$ & $4(5.5 \% ; 2.2-13.3)$ \\
Oesophagitis & $14(18.9 \% ; 11.6-29.3)$ & $21(28.8 \% ; 19.7-40)$ \\
Normal & $44(59.5 \% ; 48.1-69.9)$ & $47(64.4 \% ; 52.9-74.4)$ \\
\hline Values are number (\%; $95 \%$ confidence interval).
\end{tabular}

estimate was used for time intervals (Kaplan-Meier method). The Mantel-Cox test was used to compare $H$ pylori positive and negative subjects. A p value of $<0.05$ was considered statistically significant. Statistical analysis was performed using Bio Medical Data Processing software (BMDP Dynamic version 7.0; University of California, Los Angeles, California, USA).

\section{Ethics}

The ethics committee of St Orsola Hospital, Bologna, Italy, approved the protocol and all participants gave written informed consent.

\section{RESULTS}

A flow chart of the study is shown in fig 1 . One hundred and thirteen (66.9\% (95\% confidence interval (CI) 59.5-73.5)) subjects were infected with CagA positive strains of $\mathrm{H}$ pylori while $56 \quad(33.1 \% \quad(95 \%$ CI $26.5-40.5))$ harboured CagA negative strains of $H$ pylori. Of the 169 subjects, nine did not complete the follow up and were not included in the final analysis. Two died of colon cancer after six and eight years of follow up, respectively (one was infected with $H$ pylori in 1990). Seven refused to continue the annual follow up (one was infected with $H$ pylori in 1990). However, all of these subjects were still asymptomatic at their last follow up visit before they left the study. Of the 160 subjects who completed the study, 13 developed symptoms ( 12 H pylori positive and one $H$ pylori negative) while 147 remained asymptomatic throughout the study. Of the latter, 74 were infected with $H$ pylori (40 men and 34 women; mean (SD) age 49 (10) years) and 73 were not (47 men and 26 women; mean (SD) age 46 (11) years), as assessed by the gold standard endoscopy. Endoscopic findings are reported in table 2.

Eight (10.8\%) H pylori positive subjects who were asymptomatic (six male and two female; mean (SD) age 52 (7.7) years) had duodenal ulcers but there were no endoscopic ulcers in the $H$ pylori negative group. Fourteen subjects infected with $H$ pylori (eight male and six female; mean (SD) age 50 (7.4) years) had oesophagitis at endoscopy. All 14 patients had Los Angeles grade A oesophagitis. Five of 14 subjects were CagA positive (four male and one female;

Table 3 Endoscopic findings in the 13 blood donors who became symptomatic between 1990 and 2000 $(n=13)$

\begin{tabular}{lll}
\hline Finding & $\begin{array}{l}\boldsymbol{H} \text { pylori positive } \\
(\mathbf{n}=12)\end{array}$ & $\begin{array}{l}\boldsymbol{H} \text { pylori eradicated } \\
(\mathbf{n = 1 )}\end{array}$ \\
\hline Normal & $4(33.3 \% ; 13.8-60.9)$ & 0 \\
Duodenal ulcer & $1(8.3 \% ; 1.5-35.4)$ & 0 \\
Gastric ulcer & $1(8.3 \% ; 1.5-35.4)$ & 0 \\
Erosive duodenitis & $1(8.3 \% ; 1.5-35.4)$ & 0 \\
Erosive gastritis & $4(33.3 \% ; 13.8-60.9)$ & 0 \\
Oesophagitis & $1^{*}(8.3 \% ; 1.5-35.4)$ & $1^{*}(100 \% ; 20.7-100)$ \\
\hline
\end{tabular}

Values are number (\%; 95\% confidence interval).

*Grade A according to Los Angeles classification.

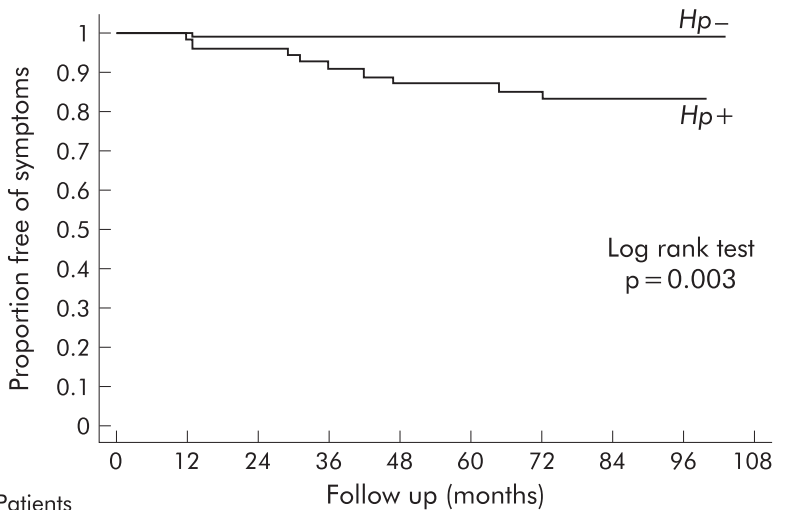

Patients

at risk

$\begin{array}{llllllllll}H p- & 81 & 81 & 80 & 80 & 79 & 79 & 79 & 78 & 14\end{array}$

$\begin{array}{llllllllll}H p+ & 88 & 86 & 85 & 81 & 79 & 78 & 76 & 76 & 16\end{array}$

Figure 2 Life table analysis of symptom development in Helicobacter pylori infected $(\mathrm{Hp+})$ asymptomatic subjects compared with those asymptomatic $H$ pylori eradicated $(\mathrm{Hp}-$ ) subjects. The former were at higher risk for the development of symptoms $(p=0.003)$.

mean (SD) age 49.4 (7.4) years). Twenty one $H$ pylori negative subjects ( 14 male and 7 female; mean (SD) age 47 (10) years) had oesophagitis; 19 subjects had grade A oesophagitis, one grade $\mathrm{B}$, and one grade $\mathrm{C}$ oesophagitis. Persistent $H$ pylori infection was not associated with a reduced risk of oesophagitis (odds ratio (OR) 0.57 (95\% CI $0.26-1.24$ ); $\mathrm{p}=0.163$ ). Among subjects with persistent $H$ pylori infection, those harbouring CagA positive strains did not have an increased risk of oesophagitis (OR 1.68 (95\% CI 0.5-5.62); $p=0.55)$. Moreover, the risk of oesophagitis in subjects infected with CagA strains compared with those not infected was not significant (OR 2.32 (95\% CI 0.8-6.8); $p=0.15$ )

\section{Development of symptoms}

Symptoms developed in 12 subjects infected with $H$ pylori and in one who had successful eradication of the infection (log rank test, $\mathrm{p}=0.003$ ) (fig $\mathrm{l}$ ). The symptoms that developed were: heartburn $(n=3)$, epigastric pain $(n=8)$, heartburn and epigastric pain $(\mathrm{n}=1)$, and regurgitation $(\mathrm{n}=1)$.

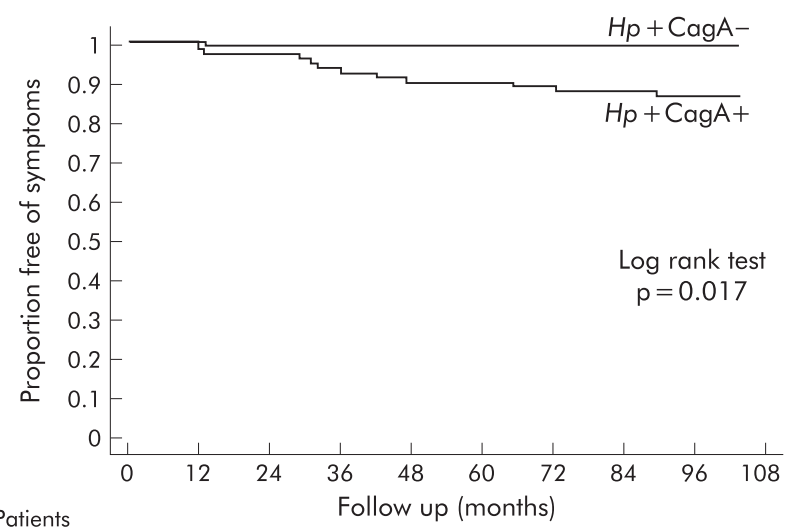

Patients

at risk

$\begin{array}{lllllllll}\mathrm{Hp}+\mathrm{CagA}-32 & 32 & 32 & 32 & 32 & 32 & 32 & 32 & 2\end{array}$

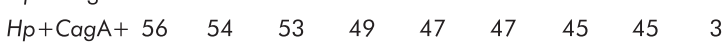

Figure 3 Life table analysis of symptom development in Helicobacter pylori CagA positive $(\mathrm{Hp}+\mathrm{CagA}+)$ infected asymptomatic subjects compared with those harbouring strains CagA negative ( $\mathrm{Hp}+\mathrm{CagA}-$ ). The former were at higher risk for the development of symptoms $(p=0.017)$. 
Endoscopic findings in subjects developing symptoms are shown in table 3. The odds ratio for the development of symptoms in H pylori positive subjects was 5.68 (95\% CI 1.8417.6) compared with those who had successful eradication. The incidence of symptoms in $H$ pylori positive subjects was 1.893/100 person-years of follow up and in $H$ pylori negative individuals $0.163 / 100$ person-years of follow up, respectively (fig 2). Subjects infected with CagA positive strains $(n=9)$ were also significantly more likely to develop symptoms compared with those who were CagA negative $(\mathrm{n}=1)$ ( $\log$ rank test, $p=0.017$ ) (fig 3 ). The odds ratio for the development of symptoms in $H$ pylori CagA positive subjects was 5.64 (95\% CI 1.36-23.5) compared with those infected with CagA negative strains.

\section{DISCUSSION}

The association between chronic $H$ pylori infection and dyspeptic symptoms is still not clear. Some population surveys report no relationship between $H$ pylori infection and dyspepsia after controlling for confounding factors. ${ }^{16-18}$ However, a large population study has recently demonstrated that $H$ pylori infection was significantly associated with dyspepsia and may be responsible for $5 \%$ of upper gastrointestinal symptoms in the community after controlling for confounding factors. ${ }^{19}$ The results of our study appear to confirm these data. We estimate that the incidence of symptoms in $H$ pylori positive subjects was $1.893 / 100$ person-years of follow up while in subjects with eradication of $H$ pylori the incidence was $0.163 / 100$ person-years of follow up. Furthermore, subjects infected with $H$ pylori CagA positive strains were more likely to develop symptoms compared with those harbouring $H$ pylori positive CagA negative strains. However, neither $H$ pylori infection nor CagA status was associated with any particular symptoms. Our data also provide an estimate of the risk of developing peptic ulcer disease. Eight of 147 subjects were found to have asymptomatic duodenal ulcers and all were still infected with $H$ pylori. We found that peptic ulcer disease was equal or more common in asymptomatic $H$ pylori infected subjects than in symptomatic $H$ pylori infected subjects. Similar findings were reported by Buckley et al who found no significant difference between the $H$ pylori positive asymptomatic group and $H$ pylori positive dyspeptic patients with regard to duodenal ulceration or antral ulceration and/or duodenal erosions. ${ }^{20}$ Population based studies have also suggested that $H$ pylori infection is a strong risk factor for ulcer disease. ${ }^{21}$

Some studies have suggested that eradication of $H$ pylori may be associated with the development of reflux oesophagitis and it has been proposed that subjects infected with CagA positive strains of $H$ pylori might be at decreased risk for GORD and its complications..$^{22}{ }^{23}$ Other studies have demonstrated that symptoms of heartburn improve after eradication of $H$ pylori and that the incidence of reflux disease is not increased. ${ }^{4225}$ However, this controversy has not been satisfactorily resolved and is important in clinical decision making because eradication therapy to prevent one disease (for example, gastric cancer) may increase the risk of another. The studies performed to date were either in patients with duodenal ulcer disease or in those with an established diagnosis of GORD. The results of our study do not support the notion that $H$ pylori infection protects against reflux disease. The number of subjects developing symptoms was small and a type II error given the small numbers cannot be ruled out. Given the low incidence of oesophagitis in both groups, it would be difficult to argue that the disappearance of $H$ pylori from Western populations is the major cause of the apparent increase in GORD in those populations. Therefore, the low incidence and apparent similarity between the $H$ pylori positive and negative groups over such a long period may be of clinical significance. Similarly, infection with a CagA positive strain did not protect against GORD. The outcome with regard to GORD may be different in our study of asymptomatic subjects because they may have a lower prevalence of severe corpus gastritis or may lack the other pathophysiological abnormalities that lead to reflux disease (lower oesophageal sphincter dysfunction, hiatal hernia).

Treatment of asymptomatic $H$ pylori infection is an area of interest for several reasons. Firstly, chronic $H$ pylori infection may lead to gastric atrophy and intestinal metaplasia, which are significant risk factors for the development of gastric cancer. $^{7}$ Treating the infection may arrest this harmful sequence, reducing the risk of gastric cancer. Gastric cancer is the second most common cause of death from malignancies in the world, and five year survival rates are less than $20 \%$ in most countries. ${ }^{26}$ Cost effectiveness studies have suggested that a screening and treatment strategy for $H$ pylori infection would be cost effective if the risk of developing gastric cancer were reduced by more than 30\%. ${ }^{27}$ However, concerns about testing healthy people, creating anxiety if eradication therapy fails, and the potential for the development of resistant strains due to widespread antibiotic use have prevented such a strategy from being adopted. ${ }^{28}$ Secondly, the cost of the management of dyspeptic patients is quite high, costing a health maintenance organisation $\$ 59.4$ million in the USA. ${ }^{29}$ Eradication treatment in subjects with asymptomatic infection could reduce downstream costs related to dyspepsia and peptic ulcer disease as well as the morbidity associated with these diseases. Although none of the patients in our study developed complicated ulcer disease, preventing complicated ulcer disease may be an additional advantage of eradication therapy in asymptomatic subjects.

The principal limitations of our study were that blinding was not performed and that it was not a randomised controlled trial of $H$ pylori eradication (that is, patients were not randomly assigned to eradication and follow up). Instead, subjects were randomly assigned to treatments in the original study and therefore success or failure of eradication was determined by the regimen patients were assigned to. The groups were comparable with regard to demographic characteristics and all subjects were healthy and asymptomatic who volunteered for blood donation when they were recruited. They were all Italians, living in the same community, with similar diets, smoking habits, and NSAID use. Selection bias needs to be considered when interpreting our results. It could be argued that patients who failed to have eradication in the original trials were non-compliant and that this may be a marker of a different lifestyle and other undefined characteristics that could influence the outcome of our study. We believe that this is unlikely because all subjects had high compliance rates in the original trial. Persistence of $H$ pylori infection was most likely due to the use of ineffective treatment combinations because proton pump inhibitor triple therapy was unknown at that time. Blinding of subjects to their $H$ pylori status was not considered ethical at the time this study was begun because we had limited knowledge of the consequences of persistent H pylori infection.

In conclusion, $H$ pylori eradication prevents the development of dyspeptic as well as peptic ulcer disease in healthy asymptomatic blood donors and is not associated with an increase in the incidence of symptomatic GORD.

\footnotetext{
Authors' affiliations

D Vaira, L Gatta, C Ricci, M Menegatti, M Miglioli, Department of Internal Medicine and Gastroenterology, University of Bologna, Bologna, Italy

N Vakil, University of Wisconsin, Medical School, Milwaukee, USA
} 
M Rugge, V M Russo, Department of Oncology and Surgical Sciences, University of Padova, Padova, Italy

G Leandro, IRCCS Castellana Grotte, Bari, Italy

J Holton, Department of Bacteriology, The Windeyer Institute of Medical Science, London, UK

\section{REFERENCES}

1 International Agency for Research on Cancer, World Health Organization. In: IARC monographs on the evaluation of the carcinogenic risks to humans, vol 61. Schistosomes, liver flukes and Helicobacter pylori. Lyon: IARC, 1994:177-241.

2 de Boer WA, Tytgat GNJ. Regular review: Treatment of Helicobacter pylori infection. BMJ 2000;320:31-4.

3 Labenz J, Blum AL, Bayerdorffer E, et al. Curing Helicobacter pylori infection in patients with duodenal ulcer may provoke reflux esophagitis.

Gastroenterology 1997;112:1442-7.

4 Moayyedi P, Bardhan C, Young L, et al. Helicobacter pylori eradication does not exacerbate reflux symptoms in gastroesophageal reflux disease. Gastroenterology 2001;121:1120-6.

5 Laine L, Schoenfeld P, Fennerty B. Therapy for Helicobacter pylori in non-ulcer dyspepsia. Ann Intern Med 2001;134:361-9.

6 Huang JQ, Sridhar S, Hunt RH. Role of Helicobacter pylori infection and nonsteroidal anti-inflammatory drugs in peptic ulcer disease: a meta-analysis. Lancet 2002;359:14-22.

7 Uemura N, Okamoto S, Yamamoto S, et al. Helicobacter pylori infection and the development of gastric cancer. N Engl J Med 2001;345:784-9.

8 Malfertheiner P, Megraud F, O'Morain C, et al. Current concepts in the management of $\mathrm{H}$ pylori infection - The Maastricht 2-2000 Consensus Report. Aliment Pharmacol Ther 2002;16:167-80.

9 Vaira D, Miglioli M, Mulè $P$, et al. Prevalence of peptic ulcer in Helicobacter pylori positive blood donors. Gut 1994;35:309-12.

10 Menegatti M, Vaira D, Figura N, et al. Clinical significance of Helicobacter pylori seropositivity and seronegativity in asymptomatic blood donors. Dig Dis Sci 1998:43:2542-8.

11 Stanghellini V and the Italian Dyspepsia Study Group (IDySG). Validation of a questionnaire for functional dyspepsia (FD) in a multicenter setting. Gut 1996;39:A102

12 Stanghellini V, Vaira D, Morselli Labate AM, et al, and the Italian Dyspepsia Study Group. Training improves the relationship of a questionnaire for functional dyspepsia (FD) in a multicenter setting. Gastroenterology 1997; 112:A831.

13 Stanghellini V, Vaira D, Morselli Labate AM, et al, and the Italian Dyspepsia Study Group. Diagnostic value of a validated questionnaire for dyspepsia. Gastroenterology 1998;114:A294.
14 Working Party of the European Helicobacter pylori Study Group. Guidelines for clinical trials in Helicobacter infection. Gut 1997;41(suppl 2):S10-18.

15 Covacci A, Censini S, Bugnoli M, et al. Molecular characterization of the 128$\mathrm{kDa}$ immunodominant antigens of Helicobacter pylori associated with cytotoxicity and duodenal ulcer. Proc Natl Acad Sci U S A 1993;90:5791-5.

16 Nandurkar S, Talley NJ, Xia H, et al. Dyspepsia in the community is linked to smoking and aspirin use but not to Helicobacter pylori infection. Arch Intern Med 1998;158:1427-33.

17 Rosenstock S, Kay L, Rosenstock C, et al. Relation between Helicobacter pylori infection and gastrointestinal symptoms and syndromes. Gut 1997;41:169-76.

18 Schlemper RJ, van der Werf SD, Vandenbroucke JP, et al. Nonulcer dyspepsia in a Dutch working population and Helicobacter pylori. Ulcer history as an explanation of an apparent association. Arch Intern Med 1995; 155:82-7.

19 Moayyedi P, Forman D, Braunholtz D, et al. The proportion of upper gastrointestinal symptoms in the community associated with Helicobacter pylori, lifestyle factors, and nonsteroidal anti-inflammatory drugs. Am J Gastroenterol 2000;95: 1448-55.

20 Buckley MJM, O'Shea J, Grace A, et al. A community-based study of the epidemiology of Helicobacter pylori infection and associated asymptomatic gastroduodenal pathology. Eur J Gastroenterol Hepatol 1998;10:375-9.

21 NIH Consensus Development Panel. Helicobacter pylori in peptic ulcer disease. J Am Med Assoc 1994;272:65-9.

22 Hamada $\mathrm{H}$, Haruma K, Mihara $M$, et al. High incidence of reflux esophagitis after eradication therapy for Helicobacter pylori: impacts of hiatal hernia and corpus gastritis. Aliment Pharmacol Ther 2000;14:729-35.

23 Vicari JJ, Peek RM, Falk GW, et al. The seroprevalence of cagA-positive Helicobacter pylori strains in the spectrum of gastroesophageal reflux disease. Gastroenterology 1998;115:50-7.

24 Schwizer M, Thumshirn M, Dent J, et al. Helicobacter pylori and symptomatic relapse of gastro-oesophageal reflux disease: a randomised controlled trial. Lancet 2001;357:1738-42

25 Wu JCY, Chan FKL, Wong SKH, et al. Effect of Helicobacter pylori eradication on oesophageal acid exposure in patients with reflux esophagitis. Alimen Pharmacol Ther 2002;15:545-52.

26 Patkin D, Oisani P, Ferlay J. Estimates of the worldwide incidence of 18 major cancers in 1985. In J Cancer 1993;54:594-606.

27 Fendrick AM, Chernew ME, Hirth R, et al. Clinical and economic effects of population-based Helicobacter pylori screening to prevent gastric cancer. Arch Intern Med 1999;159:142-8.

28 Forman D. Helicobacter pylori: the gastric cancer problem. Gut 1998;43(suppl 1):S33-4.

29 Levin TR, Schmittdiel JA, Kunz K, et al. Costs of acid-related disorders to a health maintenance organization. Am J Med 1997; 103:520-8. 